\title{
Subgenual cingulate cortex and personality in chimpanzees (Pan troglodytes)
}

\author{
Barbara J. Blatchley AND WILliam D. HopkinS \\ Agnes Scott College, Decatur, Georgia \\ and Yerkes National Primate Research Center, Atlanta, Georgia
}

\begin{abstract}
Animals vary in their dispositions, abilities, and moods and demonstrate characteristic behavior patterns that remain consistent across situation and time. This study describes the relationship between measures of personality in the chimpanzee and the structure of the subgenual cingulate cortex (SGCC). Measures of individual traits and personality factors (dominance, extraversion, conscientiousness, and agreeableness) and assessments of percentage of SGCC gray matter (PGM) and asymmetry taken from MRI scans were obtained for 74 chimpanzees housed at the Yerkes National Primate Research Center. PGM in the SGCC was significantly higher for females than for males and was significantly correlated with two personality factors (dominance and conscientiousness) in male apes. There was also a population-level leftward asymmetry in the SGCC. These results are discussed in terms of current models of SGCC function, which suggest that this area may play a role in the biological foundation of personality.
\end{abstract}

Researchers acknowledge that animals exhibit personalities. That is, they vary in their dispositions, abilities, and moods and demonstrate characteristic behavior patterns that remain consistent across situation and time (Clarke \& Boinski, 1995; Gosling, 2001). Personality consists of traits or patterns in an individual's emotional response to stimuli and in that individual's mood, which are at least partly genetically determined (Bouchard \& Loehlin, 2001).

The five-factor model (FFM) has proven to be both reliable and valid in human studies of personality. The FFM describes five clusters of personality traits in human primates, labeled neuroticism, extraversion, conscientiousness, agreeableness, and openness to experience (Costa \& McCrae, 1992; de Fruyt, McCrae, Szimak, \& Nagy, 2004; Goldberg, 1990).

Studies of nonhuman primates have shown that personality structure in chimpanzees is similar to that in humans, with clusters of traits described using the same terms. For instance, King and Figueredo (1997) devised the Chimpanzee Personality Questionnaire (CPQ) to assess personality characteristics in chimpanzees and found five aspects of personality in the chimpanzee that were similar to the five factors seen in humans and an additional sixth factor that they called dominance. Weiss, King, and Hopkins (2007) compared personalities of chimpanzees using the CPQ and found four factors that they labeled dominance, extraversion, conscientiousness, and agreeableness. They concluded that personality structure and development were largely consistent across settings.
The personality factors that make up the FFM are strongly related to mood and anxiety disorders in humans (Clark, 2005; Clark \& Watson, 1991; Clark, Watson, \& Mineka, 1994). Clark and Watson found that neuroticism was positively correlated with anxiety level and that extraversion was negatively correlated with measures of depression in humans. Researchers have proposed that the correlation between personality and psychopathology is the result of underlying temperament dimensions shared by both personality and psychopathology (Clark et al., 1994).

Only a few studies have examined the neurobiological correlates of individual differences in personality. For example, Haas, Omura, Constable, and Canli (2007b) showed that neuroticism correlated positively with amygdala and anterior subgenual cingulate activation during high emotional conflict trials on the word-face Stroop task. These authors also found that activation of the right lateral prefrontal cortex was positively correlated with scores on the agreeableness factor when individuals were asked to attend to fearful faces, but not when subjects viewed neutral, happy, or sad faces (Haas, Omura, Constable, \& Canli, 2007a). Several authors have found that specific personality factors (extraversion and neuroticism) were associated with the anatomy of the prefrontal cortex. For example, Wright et al. (2006) reported significant correlations between extraversion and neuroticism scores and the thickness of specific regions of the prefrontal cortex, Whittle et al. (2008) found that the volume of the anterior cingulate cortex was significantly correlated with measures of surgency (extraversion) and neuroticism in adolescents, 
and Blankstein, Chen, Mincic, McGrath, and Davis (2009) reported significant positive correlations between the volume of the anterior cingulate cortex and personality measures of extraversion and neuroticism. These authors also found a sex difference in this relationship. Females showed a positive correlation between gray matter volume in the subgenual cingulate cortex (SGCC) and neuroticism scores; the correlation in males was negative.

In the present study, we sought to examine whether variation in personality was associated with the volume and lateralization of the SGCC in a sample of captive chimpanzees. This was accomplished by combining recently published data on personality in a sample of chimpanzees housed at the Yerkes National Primate Center (Weiss et al., 2007) with measures of the SGCC from magnetic resonance images available in the same sample of apes. The SGCC is found ventral to the genu of the corpus callosum, and activity in this brain area has been linked to emotional responses in humans (Damasio, Tranel, \& Damasio, 1990; Liotti et al., 2000; Mayberg et al., 1999). Lesions of the SGCC are associated with increased hypothalamic-pituitary-adrenal (HPA) axis function (Diorio, Viau, \& Meaney, 1993; Sullivan \& Gratton, 1999) and with the absence of extinction of a conditioned emotional response (CER) in rats (Milad \& Quirk, 2002; Milad, Vidal-Gonzalez, \& Quirk, 2004). The projections of the SGCC to the hypothalamus, amygdala, periaqueductal gray, nucleus accumbens, and raphe nucleus have linked the SGCC to regulation of heart rate, blood pressure, body temperature, galvanic skin response, defensive reaction to threat, and depression (Freedman, Insel, \& Smith, 2000; Hurley, Herbert, Moga, \& Saper, 1991; Öngür, An, \& Price, 1998).

Given the links between SGCC function and emotional response, the purpose of this study was to determine whether the adjusted volume or percentage of gray matter present within the SGCC could be correlated with temperament and personality ascertained using the CPQ in a sample of captive chimpanzees. Previous research has demonstrated significant relationships between SGCC structure and function and the neuroticism factor in humans. Weiss et al. (2007) did not describe a neuroticism factor in the researchfacility-reared animals that constituted our sample. So, we hypothesized that SGCC volume, PGM, and asymmetry would be correlated with some or all of the personality factors that reflect the emotionality of the apes.

\section{METHOD}

\section{Subjects}

Magnetic resonance imaging (MRI) scans were obtained in 81 captive chimpanzees (Pan troglodytes), members of a captive colony housed at Yerkes National Primate Research Center (YNPRC) in Atlanta, Georgia. The chimpanzees (54 females and 27 males) ranged in age from 6.27 to 44.42 years $(M=20.42$ years, $S D=11.78)$. Of these 81 chimpanzees, personality and brain volume data were available for 74 individuals (50 females, 24 males). Statistical analyses were done using this subsample of 74 apes.

\section{Assessment of Personality}

In this study, we used the personality measures reported by Weiss et al. (2007). Briefly, in the Weiss et al. (2007) article, personality was assessed using the CPQ (King \& Figueredo, 1997), a 43-adjective questionnaire used by observers to rate their overall impressions of the chimpanzee's behaviors. Individual adjectives, reflecting traits in the behavior of the animals, were rated on a scale from 1 to 7 , where 1 indicated the absence of that trait and 7 indicated extremely large amounts of that trait. Weiss et al. (2007) described four personality factors in chimpanzees: dominance $\left(\mathrm{D}_{\mathrm{CH}}\right.$; described as dominant, manipulative, defiant, persistent, not gentle, not submissive, and not fearful), extraversion $\left(\mathrm{E}_{\mathrm{CH}}\right.$; active, social, not solitary, and not lazy), conscientiousness $\left(\mathrm{C}_{\mathrm{CH}}\right.$; predictable, not disorganized, not clumsy, not excitable, and not jealous), and agreeableness $\left(\mathrm{A}_{\mathrm{CH}}\right.$; sympathetic, sensitive, and intelligent). Unit-weighted factor scores were converted into $T$ scores, which have a mean of 50 and a standard deviation of 10. Table 1 in Weiss et al. (2007) shows the results of the principal components analysis of the traits and factors in the $\mathrm{CPQ}$, along with the salient loadings on each factor. The individual $T$ scores, as well as the 43-item specific ratings collected in the Weiss et al. (2007) study, were used in the analyses in this study. The CPQ has been shown to be both reliable and valid, with interrater reliabilities of the mean ratings of adjectives ranging from .11 to .62, with a mean of .40 (Weiss et al., 2007).

\section{Magnetic Resonance Image Collection and Procedure}

Magnetic resonance images were collected following standard procedures. The subjects were first immobilized by telazol injection $(2-6 \mathrm{mg} / \mathrm{kg})$, subsequently anesthetized with propofol $(10 \mathrm{mg} / \mathrm{kg} / \mathrm{h})$, following YNPRC standard procedures, and then transported to the MRI facility. The chimpanzees were anesthetized for the duration of the scan and transportation time between the home cage and imaging facility (total time, approximately $2 \mathrm{~h}$ ). The apes were placed in the scanner chamber in a supine position with their heads fitted inside the human head coil. Scan duration ranged from 40 to $80 \mathrm{~min}$ as a function of brain size. Approximately half the subjects $(n=33$, 21 females and 11 males) were scanned using a 1.5-Tesla scanner (Phillips, Model 51). The remaining chimpanzees ( $n=48,33$ females and 15 males) were scanned using a 3.0-T scanner (Siemens Trio; Siemens Medical Solutions, Malvern, PA) at the YNPRC. All of the apes were scanned at the YNPRC. Apes scanned at $1.5 \mathrm{~T}$ were imaged using a mobile MRI scanner (Alliance Imaging). Apes scanned at 3.0 T were imaged at the Yerkes Imaging Center, which is on-site at the YNPRC and became operational in 2005.

For all the chimpanzees scanned in vivo using the 1.5-T machine, T1-weighted images were collected in the transverse plane using a gradient echo protocol (pulse repetition $=19.0 \mathrm{msec}$, echo time $=$ $8.5 \mathrm{msec}$, number of signals averaged $=8$, matrix size $=256 \times$ 256). For the chimpanzees scanned using a 3.0-T scanner (Siemens Trio), T1-weighted images were collected using a three-dimensional gradient echo sequence (pulse repetition $=2,300 \mathrm{msec}$, echo time $=4.4 \mathrm{msec}$, number of signals averaged $=3$, matrix size $=$ $320 \times 320$ ).

After completing the MRI procedures, the subjects were returned to the YNPRC, temporarily housed in a single cage for $6-12 \mathrm{~h}$ to allow the effects of the anesthesia to wear off, and then returned to their home cage. The archived MRI data were transferred to a PC running Analyze 7.0 (Mayo Clinic, Mayo Foundation, Rochester, $\mathrm{MN})$ software for postimage processing.

\section{Quantification of the SGCC Volume}

Volumes of the subgenual region were traced manually using the freehand tool in Analyze 7.0, following the general procedure used in human subjects described by Bremner et al. (2002). The SGCC is the cortex inferior to the anterior portion of the corpus callosum (CC). In the midsagittal plane, lines were drawn perpendicular to the genu of the CC and the most posterior point of the rostrum (see Figure 1). These two lines delineated the anterior and posterior borders of the SGCC. Subsequently, the region between the anterior and posterior points in each hemisphere was traced on $1-\mathrm{mm}$ consecutive slices in the coronal plane. In the coronal plane, 


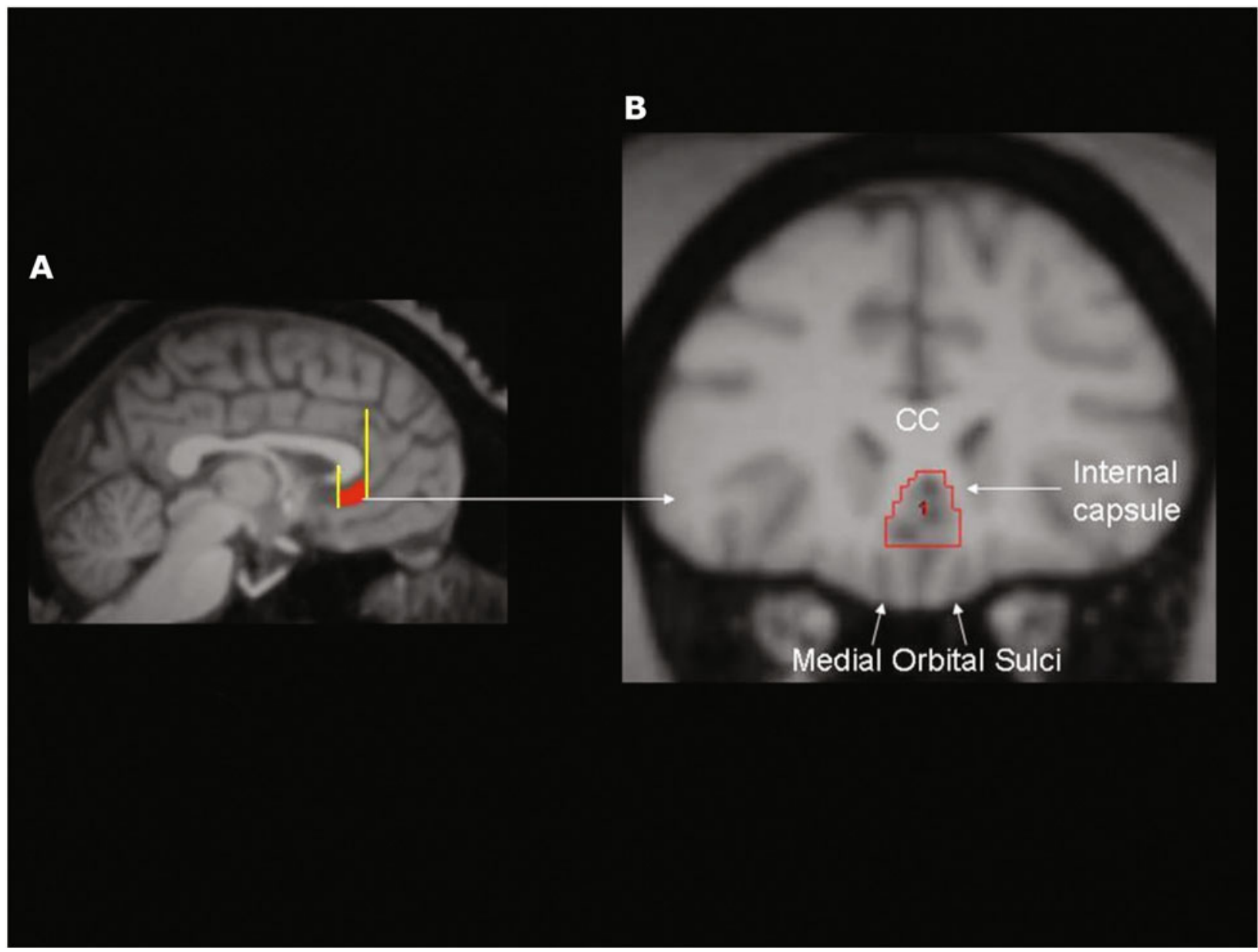

Figure 1. MRI scans showing the location of the subgenual cingulate cortex (SGCC). (A) Midsagittal section with the anterior and posterior extents of the SGCC indicated by the parallel vertical (yellow) lines and the SGCC shown in red. (B) Coronal view of the subgenual region. The borders used to define the region are labeled (see the text for a description). CC, corpus callosum.

the $\mathrm{CC}$ was the superior border, and the most dorsal projection of the left and right medial orbital sulci was the inferior border. A horizontal line was drawn between the left and right medial orbital sulci, and this served as the inferior border. The lateral border was the internal capsule. Midline was determined by finding the region of the scan where the signal-to-noise ratio was lowest. Object maps of this region were saved and subsequently applied to segmented gray matter volume to calculate the proportion of gray matter within the region.

\section{Quantification of Gray Matter Within the SGCC}

For the gray matter analysis, the T-1 MRI scans were segmented into gray, white, and CSF tissue using FSL (Analysis Group, FMRIB, Oxford, U.K.) (Smith et al. 2004). Subsequently, the object map drawn on the native scan outlining the SGCC in each hemisphere was applied to each individually aligned brain to derive volumes of gray matter found within the object maps. To determine percentage of gray matter (PGM), the volume of the gray matter was divided by the total volume and multiplied by $100\{\mathrm{PGM}=[($ Volume GM/ Total Volume $* 100]\}$ within each hemisphere. Asymmetry quotients (AQs) in the volume and gray matter were computed following the formula $[\mathrm{AQ}=(\mathrm{R}-\mathrm{L}) /(\mathrm{R}+\mathrm{L}) * .5]$, where $\mathrm{R}$ and $\mathrm{L}$ represent the total gray matter volume of the right and left hemispheres, respectively. Negative AQ values represented left-hemisphere biases, and positive values represented right-hemisphere biases.

\section{Data Analysis}

Multiple linear regression was used to evaluate the relationships between SGCC structure and personality factors and traits, controlling for age and sex. Comparisons of personality measures and PGM across sex were made using independent samples $t$ tests. For all tests, alpha was set to $p<.05$.

\section{RESULTS}

\section{Personality}

The general findings on personality for the YNPRC chimpanzees have been described by Weiss et al. (2007), and here we report some basic analyses on the subsample of apes that were used in this study. Table 1 shows the overall mean scores on the four personality factors, as well as the results of $t$ test comparisons of personality factor scores across sex. Male apes scored significantly higher on the $\mathrm{D}_{\mathrm{CH}}$ factor than did females, and females scored significantly higher on the $\mathrm{C}_{\mathrm{CH}}$ and $\mathrm{A}_{\mathrm{CH}}$ factors than did males. There were no significant sex differences in $\mathrm{E}_{\mathrm{CH}}$ scores. Studies have shown similar sex differences in personality scores in humans (McCrae, Terracciano, \& 78 
Members of the Personality Profiles of Cultures Project, 2005) and chimpanzees (King, Weiss, \& Sisco, 2008). This same pattern of results was reported by Weiss et al. (2007), and therefore, our sample of subjects reasonably represents the personality of chimpanzees that have been derived from much larger sample sizes.

\section{SGCC Gray Matter Volume and Asymmetry}

We next examined the effect of sex on SGCC gray matter volume and asymmetry in the chimpanzees. We further tested whether chimpanzees show population-level asymmetries in gray matter for the SGCC. The means and standard deviations for the PGM and AQ measures are also shown in Table 1. We found that females had significantly higher proportions of gray matter than did males $[t(72)=4.04, p<.001]$. Linear regression (sex, age, and the sex $\times$ age interaction on PGM) showed that sex was a significant predictor of $\operatorname{PGM}(\beta=-.482, p=.036)$, with sex accounting for $18.9 \%$ of the variance in the PGM measure. Age and the interaction between age and sex were not significant predictors of PGM.

We found no significant differences across sex in the AQ measure (see Table 1), and neither sex nor age was a significant predictor of AQ. The average AQ of the chimpanzees in our sample differed significantly from zero in a leftward direction, which would be the predicted value if the chimpanzees showed symmetry in the SGCC $[t(73)=$ $-5.18, p<.001]$. Fifty-four chimpanzees showed a leftward asymmetry, as compared with 20 individuals who showed a rightward asymmetry.

\section{Associations Between Personality and SGCC Gray Matter Volume and Asymmetry}

We initially performed correlations between the SGCC gray matter volume and the $T$ scores derived from the four main personality dimensions. Significant correlations were found between PGM and the $\mathrm{D}_{\mathrm{CH}}$ and $\mathrm{C}_{\mathrm{CH}}$ factors. Figure 2 shows the scatterplots of these relationships. In males, higher $\mathrm{PGM}$ was paired with higher $\mathrm{C}_{\mathrm{CH}}$ scores (see Figure $2 \mathrm{~B}$ ) and with lower $\mathrm{D}_{\mathrm{CH}}$ scores (see Figure 2A). A linear regression (age and PGM on $\mathrm{D}_{\mathrm{CH}}$ and $\mathrm{C}_{\mathrm{CH}}$ factors) showed that for males, PGM was a significant predictor of $\mathrm{D}_{\mathrm{CH}}$ and $\mathrm{C}_{\mathrm{CH}}$ factors (see Table 2). PGM accounted for $15 \%$ of the variance in $\mathrm{C}_{\mathrm{CH}}$ scores and $11 \%$ of the variance in $\mathrm{D}_{\mathrm{CH}}$ scores.

PGM was also significantly correlated with several of the individual traits that loaded onto the four personality factors, as is shown in Table 2. Apes rated as the most dominant, stingy, persistent, aggressive, defiant, reckless, and erratic tended to have the lowest PGM measures. Chimpanzees rated as highly dependent and cautious tended to have the highest PGM measures. In male apes, but not in females, linear regression (age and PGM on the individual traits that loaded onto the $\mathrm{D}_{\mathrm{CH}}$ and $\mathrm{C}_{\mathrm{CH}}$ factors) showed that PGM was a significant predictor of the traits labeled aggressive $(b=-.487, p=.016)$ and reckless ( $b=-.399, p=.050)$, accounting for $27.4 \%$ of the variance in aggressiveness and $22.7 \%$ of the variance in recklessness.
None of the four clusters of traits designated as personality factors was significantly correlated with asymmetry in either male or female animals. Females showed significant relationships between two of the individual traits that loaded on the $\mathrm{E}_{\mathrm{CH}}$ factor and the absolute value of asymmetry. For females, ratings of solitary were negatively correlated $[r(39)=-.373, p=.020]$, and ratings of helpful were positively correlated $[r(39)=.320, p=.047]$ with asymmetry. There were also relationships that trended toward significance for the traits labeled lazy $[r(39)=$ $-.300, p=.063]$ and depressed $[r(39)=-.310, p=$ $.054]$ and for asymmetry measures in females. In males, none of the correlations between individual traits that load onto the personality dimensions was significantly related to asymmetry.

\section{DISCUSSION}

Three main findings were revealed in this study. First, sex differences in personality and the percentage of gray matter making up the SGCC were evident in the chimpanzees. Second, the chimpanzees showed a significant population-level leftward asymmetry in the SGCC. Third, variation in the percentage of gray matter in the SGCC was associated with different dimensions of personality in chimpanzees, a finding that supported our hypothesis that SGCC volume and asymmetry measures would be correlated with personality factors and traits that reflect the animals' emotional responsiveness.

In terms of personality, an ape with low SGCC PGM would be described as a male who often initiated fights with other animals (aggressive), was menacing and antagonistic (reckless), was unconcerned about the consequences of his behavior (erratic), was unwilling to share food or territory (stingy), often continued a behavioral strategy despite opposition (persistent), and attempted to assert himself in a manner that countered the usual dominance order (defiant).

Traits have a genetic and physiological basis (Bouchard \& Loehlin, 2001; van Oers, de Jong, van Noordwijk, Kempenaers, \& Drent, 2005; Weiss, King, \& Figueredo, 2000), reflecting the functioning of the neural system regulating emotional response to events in the environment. The SGCC has been suggested as part of this emotional regulatory system. Several studies have reported that reduced gray matter volume and, consequently, reduced function within the subgenual prefrontal cortex are linked to disordered emotional responses (Bremner et al., 2002; Coryell, Nopoulos, Drevets, Wilson, \& Andreasen, 2005; Damasio et al., 1990; Drevets et al., 1997). The SGCC has been shown to be activated during sadness (Gemar, Kapur, Segal, Brown, \& Houle, 1997; George et al., 1995; Liotti et al., 2000), suggesting that the SGCC is specifically involved in the feeling of negative emotions. In addition, SGCC lesions result in dysregulation of the HPA axis in response to stressors (Diorio et al., 1993; Johansen-Berg et al., 2008; Sullivan \& Gratton, 1999).

In females, the lower levels of aggressive and dominant behavior patterns and higher ratings on traits as- 

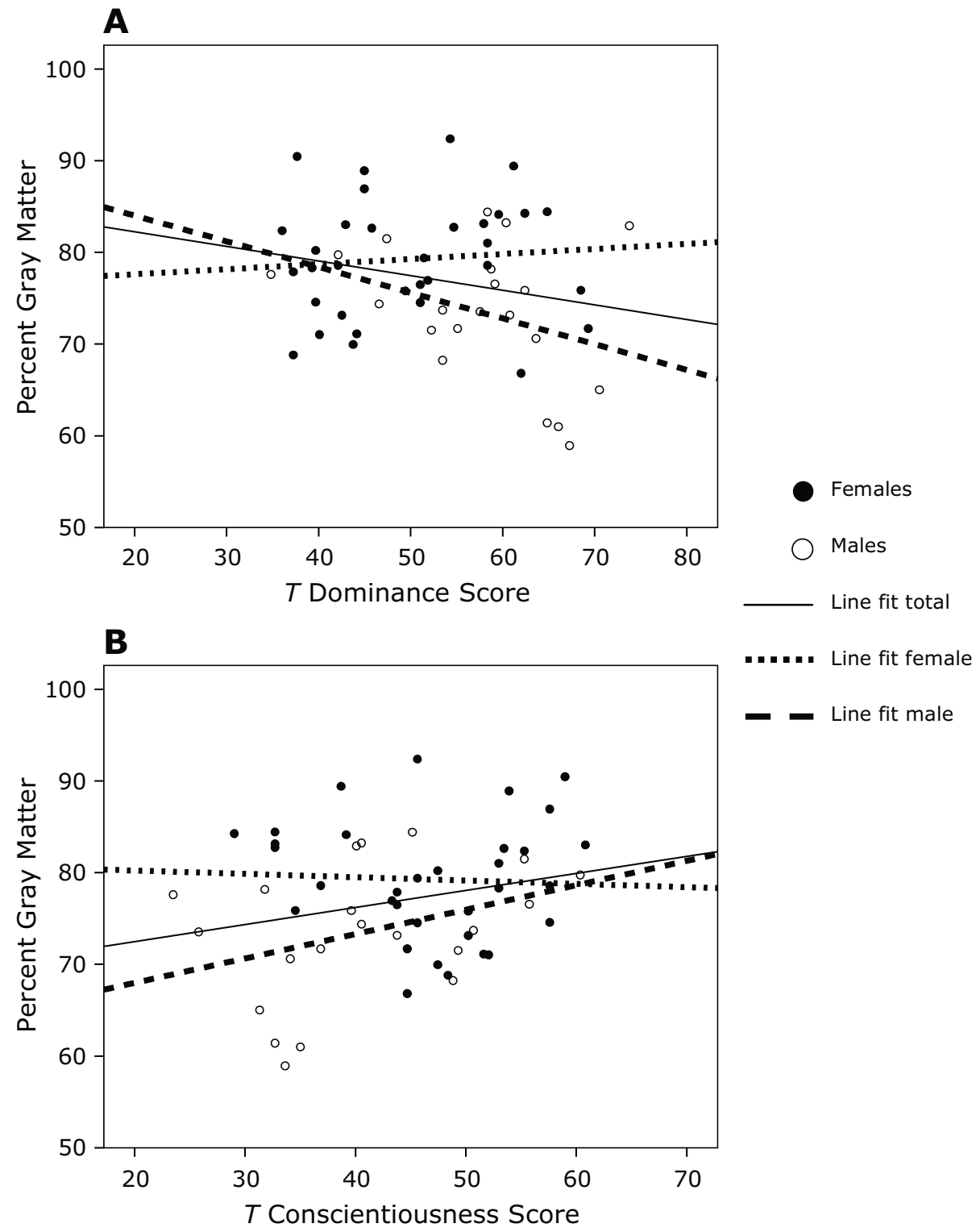

Figure 2. Scatterplots showing the relationship between dominance $\left(\mathrm{D}_{\mathrm{CH}}\right.$; panel $\left.\mathrm{A}\right)$ and conscientiousness $\left(\mathrm{C}_{\mathrm{CH}}\right.$; panel $\left.\mathrm{B}\right)$ scores and the percentage of gray matter in the subgenual cingulate cortex (SGCC). For males, high scores on the $\mathrm{C}_{\mathrm{CH}}$ factor were paired with a greater percentage of SGCC gray matter, whereas high scores on the $\mathrm{D}_{\mathrm{CH}}$ factor were paired with a smaller percentage of gray matter within the SGCC.

sociated with $\mathrm{C}_{\mathrm{CH}}$ were paired with a higher percentage of SGCC gray matter and, presumably, a higher level of function in this area of the frontal lobe. For males, more dominance, aggression, and recklessness were paired with a proportionally smaller SGCC, suggesting lower levels of function within the SGCC. Lower PGM and, presumably, reduced activity in the SGCC in our sample might also be associated with hyperactivity in the HPA and, perhaps, with a tendency to overreact to a stressor, because the ability to evaluate the consequences of a behavior depends on visceral feedback via the HPA axis and related cortical areas such as the SGCC (Damasio et al., 1990).
We found a sex difference in the relationship between the percentage of gray matter within the SGCC and personality assessment. Males scored significantly higher on the $\mathrm{D}_{\mathrm{CH}}$ factor than did females, and females scored higher on both the $\mathrm{C}_{\mathrm{CH}}$ and $\mathrm{A}_{\mathrm{CH}}$ factors than did males. In addition, $\mathrm{PGM}$ significantly predicted two traits associated with $\mathrm{D}_{\mathrm{CH}}$ and $\mathrm{C}_{\mathrm{CH}}$ in males, but not in females. Öngür et al. (1998) noted a sex difference in the projection of neurons in the SGCC in the macaque. These authors saw labeling in the ventromedial hypothalamus $(\mathrm{VMH})$ in a single female and very little VMH labeling in 3 males. They speculated that there was a sexually dimorphic projection pattern, because the VMH contains 
Table 1

Means and Standard Deviations for the Four Personality Factors, PGM, and Asymmetry Measures

\begin{tabular}{|c|c|c|c|c|c|c|c|}
\hline \multirow[b]{2}{*}{ Factor } & \multicolumn{2}{|c|}{ Overall } & \multicolumn{2}{|c|}{ Female } & \multicolumn{2}{|c|}{ Male } & \multirow[b]{2}{*}{$t$} \\
\hline & $M$ & $S D$ & $M$ & $S D$ & $M$ & $S D$ & \\
\hline $\mathrm{D}_{\mathrm{CH}}$ & 52.69 & 10.28 & 48.16 & 9.16 & 57.97 & 8.89 & $-4.33^{* *}$ \\
\hline $\mathrm{E}_{\mathrm{CH}}$ & 46.23 & 8.23 & 45.78 & 9.40 & 47.17 & 9.40 & -0.641 \\
\hline $\mathrm{C}_{\mathrm{CH}}$ & 44.71 & 9.72 & 49.25 & 9.69 & 41.99 & 10.45 & $3.11^{* *}$ \\
\hline $\mathrm{A}_{\mathrm{CH}}$ & 45.48 & 7.42 & 48.49 & 7.73 & 42.35 & 8.49 & $3.06^{* *}$ \\
\hline PGM (\%) & 77.02 & 7.37 & 79.27 & 6.52 & 73.49 & 7.39 & $4.50^{* *}$ \\
\hline AQ & -.058 & .097 & -.061 & .0975 & -.053 & .0970 & -0.339 \\
\hline
\end{tabular}

Note $-\mathrm{D}_{\mathrm{CH}}$, dominance; $\mathrm{E}_{\mathrm{CH}}$, extraversion; $\mathrm{C}_{\mathrm{CH}}$, conscientiousness; $\mathrm{A}_{\mathrm{CH}}$, agreeableness; PGM, percentage of gray matter; AQ, asymmetry quotient. ${ }^{* *} p<.01$.

sex steroid receptors and plays a role in sexual behaviors that differ across the sexes. The sex difference we found in PGM within the SGCC might be related to the extent of the projection to the hypothalamus, a known target of SGCC neurons.

Although the associations between personality and asymmetry in the SGCC were not particularly strong, we did find that the chimpanzees showed a population-level leftward asymmetry in this region. As far as we know, this is the first evidence of asymmetries within the anterior cingulate region in nonhuman primates and adds to a growing body of literature documenting neuroanatomical asymmetries in nonhuman primates (see Hopkins, 2007). What the functional significance of this asymmetry is with respect to behavior is unclear from this study, but this should be addressed in future studies.

\section{Limitations of the Study}

A potential confounding variable in this study has to do with defining and locating specific cortical areas. We did not do the cytoarchitectonic analysis necessary to determine whether the region we measured corresponded to Brodmann's Area 25, the specific subregion of the subgenual cingulate linked to a pattern of negative emotional reactions characteristic of clinical depression. Brodmann originally included all of the cortex ventral to the genu of the CC in Area 24. He reevaluated his organizational schema in 1909, separating Area 25; however, there is still some disagreement over the exact boundaries of Area 25 in the literature. For example, Drevets, Öngür, and Price (1998, p. 223) disagreed with the description of this area used by Mayberg et al. (1997, as cited in Öngür et al., 1998) stating that "Mayberg, et al., referred to this area as Brodmann Area 25, although in the human brain the cingulate cortex in this position corresponds primarily to Area 24b, and Area 25 (i.e., the infralimbic cortex) instead lies more caudal."

The region we measured may have included Brodmann's Area 25, as well as other subregions. However, the evidence that the cortex ventral to the genu of the $\mathrm{CC}$ in the anterior cingulate is involved in the regulation of behavioral responses to emotional stimuli seems powerful and persuasive. Our study links this area to personality and temperament in nonhuman primates and suggests future research on individual differences in vulnerability to depression.

It should also be noted that GM volumes were derived from MRI scans obtained on different magnets (1.5 vs. 3.0 T). Differences in magnet strength can influence the contrast in gray-white matter in the scans and lead to differences in GM volumes. There was a significant difference in the volume of gray matter measures. There was a significant difference in the PGM measures obtained using different magnets $[F(1,77)=4.14, p<.05]$. To correct for the magnet used, gray matter volumes were converted to standardized $z$ scores, and the correlations with personality factors were recalculated. Standardized GM volumes were significantly and positively corre-

Table 2

Multiple Regression Analysis Predicting Personality Factors and Traits From Percentage of Gray Matter (PGM) in the SGCC

\begin{tabular}{|c|c|c|c|c|c|c|}
\hline Factor & $\begin{array}{l}\text { Correlation } \\
\text { With PGM: } \\
\text { All Animals }\end{array}$ & $\begin{array}{c}\beta: \text { All } \\
\text { Animals }\end{array}$ & Trait & $\begin{array}{c}\text { Loads Onto } \\
\text { Factor } \\
\text { (Positively or } \\
\text { Negatively) }\end{array}$ & $\begin{array}{l}\text { Correlation } \\
\text { With PGM: } \\
\text { All Animals }\end{array}$ & $\begin{array}{c}\beta \text { : Males } \\
\text { Only }\end{array}$ \\
\hline $\mathrm{D}_{\mathrm{CH}}$ & $-.281^{* *}$ & $-.277^{* *}$ & $\begin{array}{l}\text { dominant } \\
\text { stingy } \\
\text { persistent } \\
\text { dependent } \\
\text { cautious }\end{array}$ & $\begin{array}{l}\mathrm{P} \\
\mathrm{P} \\
\mathrm{P} \\
\mathrm{N} \\
\mathrm{N}\end{array}$ & $\begin{array}{c}-.274^{*} \\
-.269^{*} \\
-.240^{*} \\
.248^{*} \\
.247^{*}\end{array}$ & $\begin{array}{l}-.112 \\
-.270^{*} \\
-.221 \\
.029 \\
.050\end{array}$ \\
\hline $\mathrm{C}_{\mathrm{CH}}$ & $.326^{* *}$ & $.321^{* *}$ & $\begin{array}{l}\text { aggressive } \\
\text { defiant } \\
\text { reckless } \\
\text { erratic }\end{array}$ & $\begin{array}{l}\mathrm{N} \\
\mathrm{N} \\
\mathrm{N} \\
\mathrm{N}\end{array}$ & $\begin{array}{l}-.394^{* *} \\
-.317^{* *} \\
-.285^{* *} \\
-.247^{*}\end{array}$ & $\begin{array}{l}-.225^{*} \\
-.291^{*} \\
-.150 \\
-.112\end{array}$ \\
\hline
\end{tabular}

Note $-\mathrm{D}_{\mathrm{CH}}$, dominance; $\mathrm{C}_{\mathrm{CH}}$, conscientiousness. $\quad{ }^{*} p \leq .05 . \quad{ }^{* *} p \leq .01$. 
lated with the $\mathrm{D}_{\mathrm{CH}}$ factors and significantly and negatively correlated with the $\mathrm{C}_{\mathrm{CH}}$ factors, as was found in the original analysis.

More work is needed to describe the role of the SGCC in the regulation of emotional response. However, the results of this study support the hypothesis that forebrain systems, including the SGCC, are correlated with personality traits and factors that reflect the animals' emotional responsiveness.

\section{AUTHOR NOTE}

This research was supported in part by NIH Grants NS-42867, HD-38051, and HD-56232. The Yerkes Center is fully accredited by the American Association for Accreditation of Laboratory Animal Care. American Psychological Association guidelines for the ethical treatment of animals were adhered to during all aspects of this study. Correspondence concerning this article should be addressed to B. J. Blatchley, Department of Psychology, Agnes Scott College, 141 E. College Avenue, Decatur, GA 30030 (e-mail: bblatchley@agnesscott.edu).

\section{REFERENCES}

Blankstein, U., Chen, J. Y. W., Mincic, A. M., McGrath, P. A., \& Davis, K. D. (2009). The complex minds of teenagers: Neuroanatomy of personality differs between sexes. Neuropsychologia, 47, 599-603.

Bouchard, T. J., JR., \& Loehlin, J. C. (2001). Genes, evolution, and personality. Behavior Genetics, 31, 243-273.

Bremner, J. D., Vythilingam, M., Vermetten, E., Nazeer, A., Adil, J., Staib, L. H., \& Charney, D. S. (2002). Reduced volume of orbitofrontal cortex in major depression. Biological Psychiatry, 51, 273-279.

Clark, L. A. (2005). Temperament as a unifying basis of personality and psychopathology. Journal of Abnormal Psychology, 114, 505-521.

Clark, L. A., \& Watson, D. (1991). Tripartite model of anxiety and depression: Psychometric evidence and taxonomic implications. Journal of Abnormal Psychology, 100, 316-336.

Clark, L. A., Watson, D., \& MineKa, S. (1994). Temperament, personality and the mood and anxiety disorders. Journal of Abnormal Psychology, 103, 103-116.

Clarke, S. A., \& Boinski, S. (1995). Temperament in nonhuman primates. American Journal of Primatology, 37, 103-125.

Coryell, W., Nopoulos, P., Drevets, W., Wilson, T., \& Andreasen, N. C. (2005). Subgenual prefrontal cortex volumes in major depressive disorder and schizophrenia: Diagnostic specificity and prognostic implications. American Journal of Psychiatry, 162, 1706-1712.

Costa, P. T., \& McCrae, R. R. (1992). Revised NEO Personality Inventory (NEO-PI-R) and NEO Five-Factor Inventory (NEO-FFI) professional manual. Odessa, FL: Psychological Assessment Resources.

Damasio, A. R., Tranel, D., \& Damasio, H. (1990). Individuals with sociopathic behavior caused by frontal damage fail to respond autonomically to social stimuli. Behavioural Brain Research, 41, 81-94.

de Fruyt, F., McCrae, R. R., SzimaK, Z., \& Nagy, J. (2004). The FiveFactor Personality Inventory as a measure of the five-factor model. Belgian, American, and Hungarian comparison with the NEO-PI-R. Assessment, 11, 207-215.

Diorio, D., Viau, V., \& Meaney, M. J. (1993). The role of the medial prefrontal cortex (cingulate gyrus) in the regulation of the hypothalamic-pituitary-adrenal response to stress. Journal of Neuroscience, 13, 3839-3847.

Drevets, W. C., ÖNGÜR, D., \& PrICE, J. L. (1998). Neuroimaging abnormalities in the subgenual prefrontal cortex: Implications for the pathophysiology of familial mood disorders. Molecular Psychiatry, 3, 220-226.

Drevets, W. C., Price, J. L., Simpson, J. R., Todd, R. D., Reich, T., VANNIER, M., \& RAICHLE, M. E. (1997). Subgenual prefrontal cortex abnormalities in mood disorders. Nature, 386, 824-827.

Freedman, L. J., InSEL, T. R., \& SMith, Y. (2000). Subcortical projections of Area 25 (subgenual cortex) of the macaque monkey. Journal of Comparative Neurology, 421, 172-188.

Gemar, M. C., Kapur, S., Segal, Z. V., Brown, G. M., \& Houle, S.
(1997). Effects of self-generated sad mood on regional cerebral activity: A PET study in normal subjects. Depression, 4, 81-88.

George, M. S., Ketter, T. A., Parekh, P. I., Horwitz, B., HerscoVITCH, P., \& Post, R. M. (1995). Brain activity during transient sadness and happiness in healthy women. American Journal of Psychiatry, 152, 341-351.

GoLDBERG, L. R. (1990). An alternative "description of personality": The big-five factor structure. Journal of Personality \& Social Psychology, 59, 1216-1229.

GosLing, S. D. (2001). From mice to men: What can we learn about personality from animal research? Psychological Bulletin, 127, 45-86.

Haas, B. W., Omura, K., Constable, R. T., \& Canli, T. (2007a). Emotional conflict and neuroticism: Personality dependent activation in the amygdala and subgenual anterior cingulate. Behavioral Neuroscience, 121, 249-256.

Haas, B. W., Omura, K., Constable, R. T., \& Canli, T. (2007b). Is automatic emotion regulation associated with agreeableness? A perspective using a social neuroscience approach. Psychological Science, 18, 130-132.

HoPKINS, W. D. (ED.) (2007). Evolution of hemispheric specialization in primates. London: Academic Press.

Hurley, K. M., Herbert, H., Moga, M. M., \& Saper, C. B. (1991). Efferent projections of the infralimbic cortex of the rat. Journal of Comparative Neurology, 308, 249-276.

Johansen-Berg, H., Gutman, D. A., Behrens, T. E. J., Matthews, P. M., Rushworth, M. F. S., Katz, E., ET AL. (2008). Anatomical connectivity of the subgenual cingulate region targeted with deep brain stimulation for treatment-resistant depression. Cerebral Cortex, 18, 1374-1383.

King, J. E., \& Figueredo, A. J. (1997). The five-factor model plus dominance in chimpanzee personality. Journal of Research in Personality, 31, 257-271.

King, J. E., Weiss, A., \& Sisco, M. S. (2008). Aping humans: Age and sex effects in chimpanzee (Pan troglodytes) and human (Homo sapiens) personality. Journal of Comparative Psychology, 122, 418-427.

Liotti, M., Mayberg, H. S., Brannan, S. K., McGinnis, S., JeraBEK, P., \& Fox, P. T. (2000). Differential limbic-cortical correlates of sadness and anxiety in healthy subjects: Implications for affective disorders. Biological Psychiatry, 48, 30-42.

Mayberg, H. S., Liotti, M., Brannan, S. K., McGinnis, S., JerabeK, P. A., Martin, C. C., ET AL. (1997). Disease-specific effects of mood challenge in remitted depression. Society for Neuroscience Abstracts, 23, 1406.

Mayberg, H. S., Liotti, M., Brannan, S. K., McGinnis, S., MahuRIN, R. K., JERABEK, P. A., ET AL. (1999). Reciprocal limbic-cortical function and negative mood: Converging PET findings in depression and normal sadness. American Journal of Psychiatry, 156, 675-682.

McCrae, R. R., Terracciano, A., \& 78 Members of the Personality Profiles of Cultures Project (2005). Universal features of personality traits from the observer's perspective: Data from 50 cultures. Journal of Personality \& Social Psychology, 88, 547-561.

Milad, M. R., \& QuIRK, G. J. (2002). Neurons in medial prefrontal cortex signal memory for fear extinction. Nature, 420, 70-74.

Milad, M. R., Vidal-Gonzalez, I., \& QuirK, G. J. (2004). Electrical stimulation of medial prefrontal cortex reduces conditioned fear in a temporally specific manner. Behavioral Neuroscience, 118, 389-394.

ÖNGÜR, D., AN, X., \& PrICE, J. L. (1998). Prefrontal cortical projections to the hypothalamus in macaque monkeys. Journal of Comparative Neurology, 401, 480-505.

Smith, S. M., Jenkinson, M., Woolrich, M. W., Beckmann, C. F., Behrens, T. E. J., Johansen-Berg, H., Et AL. (2004). Advances in functional and structural MR image analysis and implementation as FSL. NeuroImage, 23, S208-S219.

Sullivan, R. M., \& Gratton, A. (1999). Lateralized effects of medial prefrontal cortex lesions on neuroendocrine and autonomic stress responses in rats. Journal of Neuroscience, 19, 2834-2840.

van Oers, K., De Jong, G., van NoordwiJk, A. J., Kempenaers, B., \& Drent, P. J. (2005). Contribution of genetics to the study of animal personalities: A review of case studies. Behaviour, 142, 1191-1212.

Weiss, A., King, J. E., \& Figueredo, A. J. (2000). The heritability of personality factors in chimpanzees (Pan troglodytes). Behavioral Genetics, 30, 213-221.

Weiss, A., KING, J. E., \& Hopkins, W. (2007). A cross-setting study of 
chimpanzee (Pan troglodytes) personality structure and development: Zoological parks and Yerkes National Primate Center. American Journal of Primatology, 69, 1264-1277.

Whittle, S., Yucel, M., Fornito, A., Barrett, A., Wood, S. J., LubMAN, D. I., ET AL. (2008). Neuroanatomical correlates of temperament in early adolescents. Journal of the American Academy of Child \& Adolescent Psychiatry, 47, 682-693.
Wright, C. I., Williams, D., Feczko, E., Barrett, L. F., Dickerson, B. C., Schwartz, C. E., \& Wedig, M. M. (2006). Neuroanatomical correlates of extraversion and neuroticism. Cerebral Cortex, 16, 1809-1819.

(Manuscript received October 30, 2009; revision accepted for publication May 24, 2010.) 